\title{
Olpidium bornovanus-Mediated Germination of Ascospores of Monosporascus cannonballus: A Host-Specific Rhizosphere Interaction
}

\author{
Michael E. Stanghellini and Iraj J. Misaghi
}

Department of Plant Pathology and Microbiology, University of California, Riverside 92521. Accepted for publication 10 February 2011.

\begin{abstract}
Stanghellini, M. E., and Misaghi, I. J. 2011. Olpidium bornovanusmediated germination of ascospores of Monosporascus cannonballus: A host-specific rhizosphere interaction. Phytopathology 101:794-796.

Monosporascus cannonballus, a host-specific root-infecting ascomycete, is the causal agent of a destructive disease of melon (Cucumis melo L.) known as vine decline. Ascospores germinate only in the rhizosphere of melon plants growing in field soil. However, no germination occurs in the rhizosphere of melon plants if the field soil is

observation suggested that germination is mediated by one or more heatsensitive members of the soil microflora. Although bacteria or actinomycetes were heretofore suspected as the germination-inducing microbes, our data demonstrate that Olpidium bornovanus, an obligate, hostspecific, root-infecting zoosporic fungus, is responsible. In four experiments conducted in autoclaved field soil amended with various population densities of culturally produced ascospores, significant ascospore germination was recorded only in the rhizosphere of cantaloupe seedlings colonized by $O$. bornovanus.
\end{abstract} heated to temperatures $>50^{\circ} \mathrm{C}$ prior to infestation with ascospores. This
Monosporascus cannonballus Pollack \& Uecker $(8,15)$, an unusual root-infecting ascomycete, is recognized worldwide as the causal agent of a destructive disease of melon known as vinedecline $(3,7,13)$. Perithecia, containing single-spored asci, are produced in the cortex of infected and moribund roots during the latter part of the growing season. No other spore stage is known to be produced by this pathogen. Thus, ascospores apparently function as both the sole survival propagules and primary inoculum for this soilborne fungus (11). However, unlike survival propagules of most soilborne, root-infecting fungal and fungallike pathogens $(5,9)$, ascospores of $M$. cannonballus, either naturally or culturally produced, will not germinate in axenic culture (15) (unpublished data). Further, germination of ascospores is extremely host specific (the opposite of most soilborne pathogens) and occurs only in the rhizosphere of certain genera and species of plants belonging exclusively to the family Cucurbitaceae, with cantaloupe (Cucumis melo L.) being the most germination-stimulatory and susceptible cultivar (10). However, ascospores do not germinate in the rhizosphere of cantaloupe plants if the field soil is either autoclaved (12) or heated to temperatures $>50^{\circ} \mathrm{C}$ (M. E. Stanghellini, unpublished) prior to artificial infestation with ascospores. The heat sensitivity suggests that (i) ascospore germination is mediated, either directly or indirectly, by one or more heat-sensitive members of the soil microflora and (ii) cantaloupe root exudates alone are not the germination stimulant. Additionally, the delayed onset of ascospore germination in the rhizosphere, which requires 6 to 7 days (12), compared with 2 to $12 \mathrm{~h}$ (5) for the survival propagules of most soilborne pathogens, suggests that some factor, such as a specific microbe population, must increase to a critical threshold level before ascospore germination is induced (12).

Over the past 9 years, our studies have been focused on the isolation of soil microbes presumed to be involved in ascospore

Corresponding author: M. E. Stanghellini;

E-mail address: michael.stanghellini@ucr.edu

doi:10.1094/PHYTO-11-10-0313

(c) 2011 The American Phytopathological Society germination. Unfortunately, none of the microbes tested (which included numerous bacteria and actinomycetes isolated from bulk field soil, the rhizosphere or rhizoplane of cantaloupe plants supporting ascospore germination, or ascospore germlings physically detached from cantaloupe roots) were capable, either alone or in combination at various population densities, of inducing ascospore germination in the rhizosphere of cantaloupe growing in autoclaved soil (M. E. Stanghellini, unpublished data).

Recently, we reported on the pathogenicity of a melon strain of Olpidium bornovanus on greenhouse-grown melon plants (14). This obligate, root-infecting holocarpic fungus is host specific to cantaloupe (2) and our preliminary studies showed that the melon strain of $O$. bornovanus is common in soil samples collected from commercial cantaloupe fields with a history of vine decline caused by M. cannonballus in California and Arizona (14). Thus, our objective was to test the possible involvement of $O$. bornovanus in the induction of ascospore germination in field soil.

\section{MATERIALS AND METHODS}

Host and pathogen. All experiments were conducted using cantaloupe (C. melo L. 'Caravelle') as the susceptible host.

Two single sporangium isolates of $O$. bornovanus (IP-1 and $\mathrm{H}-6)$, one recovered from soil from melon fields in the Imperial Valley, CA and the other from the Harquahala Valley, AZ, respectively, were separately maintained on cantaloupe seedlings growing in vermiculite in a growth chamber. Roots of seedlings bearing numerous sporangia served as the source of zoospores for inoculation of cantaloupe seedlings. Zoospores were released from sporangia within $5 \mathrm{~min}$ after colonized roots were excised from the source plants and incubated in $15 \mathrm{ml}$ of tap water in 25-ml glass beakers.

Two single-ascospore isolates of M. cannonballus (BH3-19 and Mc14), obtained from infected roots of cantaloupe plants from a commercial melon field in Arizona and California, respectively, and separately maintained on V8 agar medium, were used for infestation of field soil.

Soil infestation procedure. A previously described method for infesting field soil with populations of culturally produced asco- 
spores was used (12). Briefly, autoclaved volumes of a melon field soil (Meloland sandy loam) were infested with ascospores recovered from 1-month-old V8 agar cultures of M. cannonballus. Three experiments, involving soil volumes artificially infested with three different population densities of ascospores, were conducted using M. cannonballus isolate BH3-19 and O. bornovanus isolate IP-1. Ascospore population densities in the three separate soil volumes were estimated (11) at $430 \pm 25,2,100 \pm$ 300 , and 5,000 \pm 425 ascospores $\mathrm{g}^{-1}$ of soil. Additionally, one experiment was conducted using $O$. bornovanus isolate $\mathrm{H}-6$ and M. cannonballus isolate Mc14. The ascospore population in the latter infested soil was $300 \pm 22$ ascospores $\mathrm{g}^{-1}$ of soil. The ascospore-infested soils was air dried and stored in plastic bags at $24^{\circ} \mathrm{C}$ until used.

Ascospore germination assessment. A previously described method for assessing ascospore germination was used $(11,12)$.

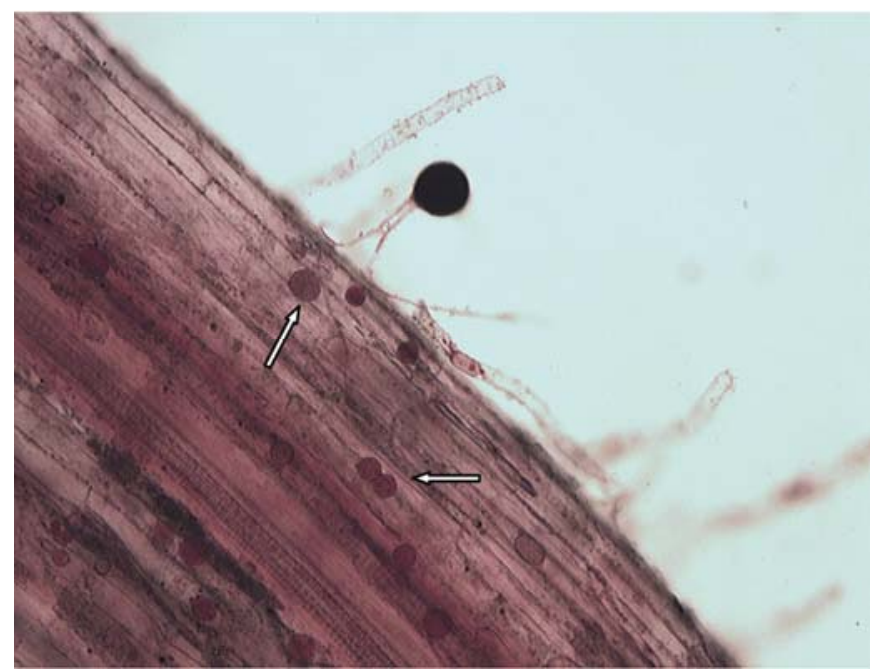

Fig. 1. Germinated ascospore of Monosporascus cannonballus in close proximity to sporangia of Olpidium bornovanus (arrows) in epidermal cells of a cantaloupe root. Ascospore diameter $=45 \mu \mathrm{m}$.
Subsamples of autoclaved field soil amended with ascospores of $M$. cannonballus were dispensed into polyethylene centrifuge tubes $(5 \mathrm{~cm}$ long and $1.7 \mathrm{~cm}$ in diameter) with drainage holes drilled through the bottom of each tube. One 3-day-old cantaloupe seedling was transplanted into each tube. Radical length of the pregerminated seedlings (healthy or inoculated with zoospores of $O$. bornovanus) was 2.5 to $3.0 \mathrm{~cm}$ at transplant. Inoculation of cantaloupe seedlings with zoospores of $O$. bornovanus was accomplished as follows: radicals of pregerminated seedlings were placed for $10 \mathrm{~min}$ in 10 -ml vials containing $8 \mathrm{ml}$ of sterile distilled water containing $\approx 300$ zoospores of $O$. bornovanus $/ \mathrm{ml}$. Our preliminary studies showed that higher concentrations of zoospores frequently resulted in seedling death within 5 to 7 days after inoculation (unpublished data). Inoculated seedlings were then transplanted into the tubes and placed in a growth chamber at $30^{\circ} \mathrm{C}$ with a 12 -h photoperiod. The seedlings were irrigated with sterile distilled water every other day. After 9 days of incubation, seedlings were removed from the tubes and placed in petri dishes containing sterile distilled water and observed with a dissecting and compound microscope. The number of ascospore germlings attached to the root system of each plant was recorded.

Statistical analysis. All experiments were replicated three times. The unpaired Student's $t$ test was performed to determine whether the number of ascospore germlings attached to plant roots inoculated with $O$. bornovanus was significantly different $(P \leq 0.05)$ from plant roots that were not inoculated with $O$. bornovanus.

\section{RESULTS}

Ascospore germination assessment in autoclaved soil. Sporangia of $O$. bornovanus, in various stages of maturation, were microscopically observed in epidermal cells of roots of all Olpidium-inoculated seedlings (Fig. 1). No sporangia were observed in roots of cantaloupe seedlings that were not inoculated with $O$. bornovanus. In all four experiments, the number of ascospore germlings of $M$. cannonballus attached to roots of 9-day-old cantaloupe seedlings which were inoculated with zoospores of $O$. bornovanus prior to transplant was significantly

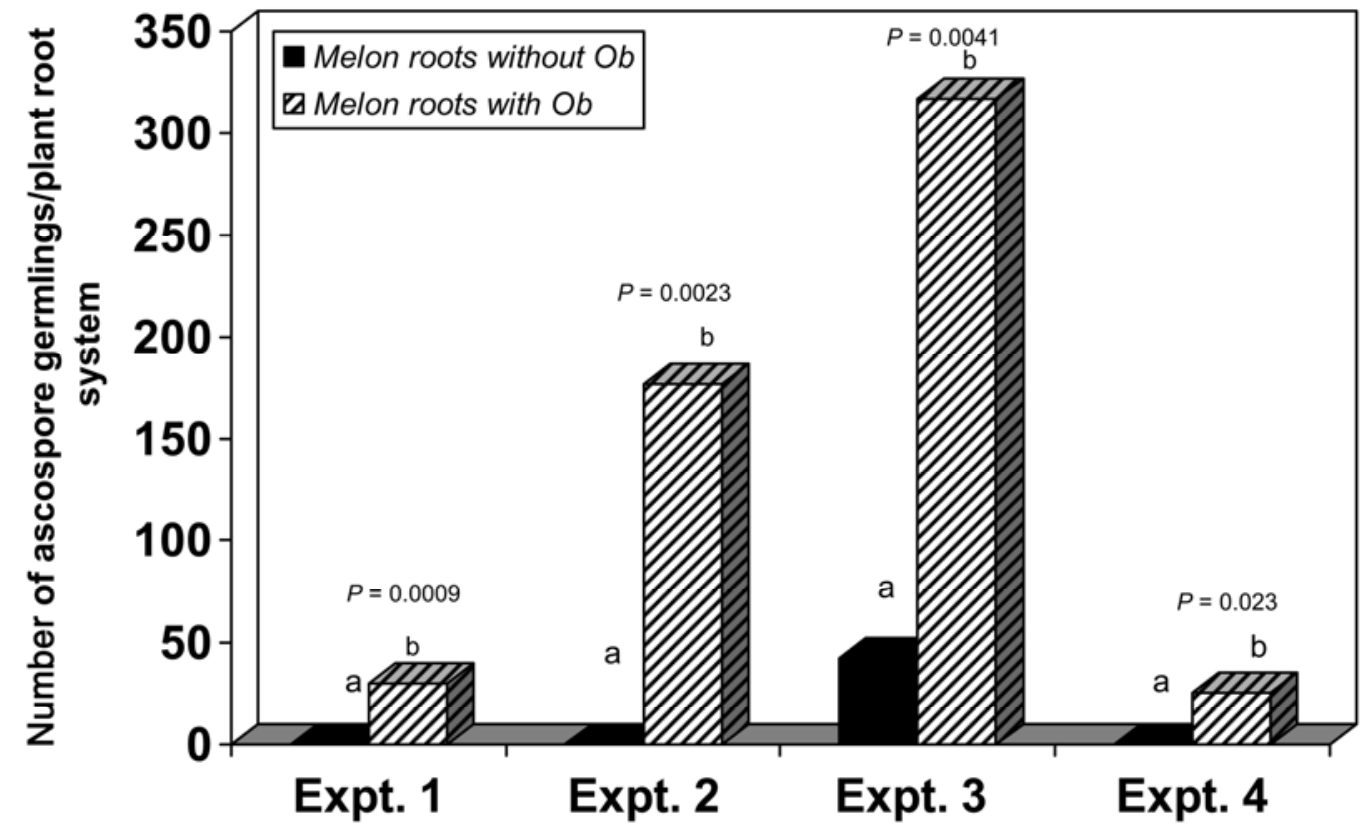

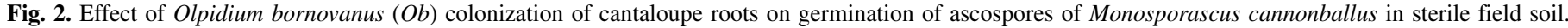

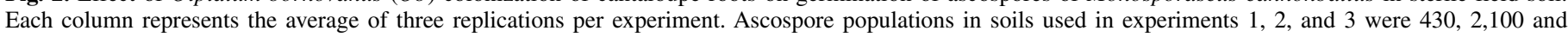

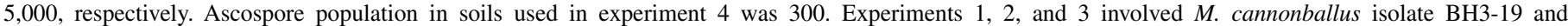

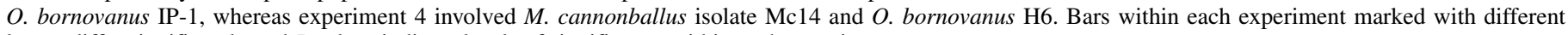
letters differ significantly and $P$ values indicate levels of significance within each experiment. 
higher $(P \leq 0.05)$ compared with seedlings that were not inoculated with $O$. bornovanus (Fig. 2).

\section{DISCUSSION}

After nearly a decade of numerous unsuccessful experiments by colleagues in our laboratory to identify the microbe or microbes involved in the tripartite rhizosphere interaction required for the induction of germination of ascospores of $M$. cannonballus, a highly probable candidate has finally emerged. Both direct and indirect evidence suggest that $O$. bornovanus, an obligate, host-specific, root-infecting, and pathogenic fungus, is associated with the induction of ascospore germination.

First, the direct evidence. Significant ascospore germination in sterile field soil occurred only in the rhizosphere of cantaloupe roots that were colonized by $O$. bornovanus. This response was verified in four separate experiments involving two isolates of $O$. bornovanus (one from Arizona and one from California) and in a soil artificially infested with four different ascospore populations of two isolates of $M$. cannonballus (one from Arizona and one from California). However, a number of ascospore germlings were observed on cantaloupe roots that were not colonized by $O$. bornovanus in one experiment (experiment 3). A likely explanation for this apparent anomaly could be that a low percentage of culturally produced ascospores are physiologically capable of germination. The soil in experiment 3 was infested with a high population of ascospores $\left(5,000 \pm 425\right.$ ascospores $\mathrm{g}^{-1}$ of soil).

Second, the indirect evidence. The melon strain of $O$. bornovanus (2), like $M$. cannonballus (10), is host specific (only the cucurbit family), with the same preference for cantaloupe $(2,10)$, and the cantaloupe strain of $O$. bornovanus has been consistently recovered from melon field soils with a known history of vine decline caused by $M$. cannonballus (14). Further, we previously noted that ascospores of $M$. cannonballus require an incubation period 6 to 9 days in the rhizosphere before the onset of germination $(11,12)$. This time frame coincides with the maturation rate of sporangia of $O$. bornovanus following zoospore infection of epidermal cells of a cantaloupe root (14).

Cumulatively, both direct and indirect evidence supports our conclusion that $O$. bornovanus is directly involved in the induction of germination of ascospores of $M$. cannonballus in field soil. $O$. bornovanus may not be the only soil microbes, however, with that capability but we have not, to date, been able to identify or implicate any other microbe.

Although beneficial tripartite rhizosphere interactions (i.e., legume-AM fungi-rhizobia) (1) have been described, the detrimental tripartite rhizosphere interaction reported herein is unique. To our knowledge, only one other root-infecting pathogen ex- hibits a similar host-specific tripartite interaction (i.e., Sclerotium cepivorum) (4). In soil, sclerotia of S. cepivorum are stimulated to germinate only in the rhizosphere of members of the genus Allium. Specific Allium spp. root exudates (e.g., the water-soluble, nonvolatile allyl cysteine sulphoxides) are broken down by unidentified soil bacteria to give mixtures of volatile alkyl thiols and sulfides (6). These degradation products apparently function as the stimulus for germination of sclerotia in field soil. However, the specific mechanisms which induce ascospore germination in field soil are not known.

\section{LITERATUE CITED}

1. Barea, J.-M., Pozo, M. J., Azcón, R., and Azcón-Aguilar, C.. 2005. Microbial co-operation in the rhizosphere. J. Exp. Bot. 56:1761-1778.

2. Campbell, R. N., and Sim, S. T. 1994. Host specificity and nomenclature of Olpidium bornovanus (= Olpidium radicale) and comparisons to Olpidium brassicae. Can J. Bot. 72:1136-1143.

3. Cohen, R., Pivonia, S., Burger, Y., Edelstein, M., Gamliel, A., and Katan, J. 2000. Toward integrated management of Monosporascus wilt of melons in Israel. Plant Dis. 84:496-505.

4. Coley-Smith J. R., Dickinson, D. J., King, J. E., and Holt, R. W. 1968. The effect of species of Allium on soil bacteria in relation to germination of sclerotia of Sclerotium cepivorum. Ann. Appl. Biol. 62:103-111.

5. Huisman, O. C. 1982. Interrelations of root growth dynamics to epidemiology of root-invading fungi. Annu. Rev. Phytopathol. 20:303-327.

6. King, J. E., and Colely-Smith, J. R. 1969. Effects of volatile products of Allium species and their extracts on germination of Sclerotium cepivorum Berk. Ann. Appl. Biol. 64:303-314.

7. Martyn, R. D., and Miller, M. E. 1996. Monosporascus root rot and vine decline: An emerging disease of melons worldwide. Plant Dis. 80:716725 .

8. Pollack, F. G., and Uecker, F. A. 1974. Monosporascus cannonballus, an unusual ascomycete in cantaloupe roots. Mycologia 66:346-349.

9. Schroth, M. N., and Hildebrand, D. C. 1964. Influence of plant root exudates on root- infecting fungi. Annu. Rev. Phytopathol. 2:101-132.

10. Stanghellini, M. E., Alcantara, T. P., and Ferrin, D. M. 2010. Germination of Monosporascus cannonballus ascospores in the rhizosphere: a hostspecific response. Can. J. Plant Pathol. 32:402-405.

11. Stanghellini, M. E., Kim, D. H., and Rasmussen, S. L. 1996. Ascospores of Monosporascus cannonballus: germination and distribution in cultivated and desert soils. Phytopathology 86:509-514.

12. Stanghellini, M. E., Kim, D. H., and Waugh, M. 2000. Microbe-mediated germination of ascospores of Monosporascus cannonballus. Phytopathology 90:243-247.

13. Stanghellini, M. E., Kim, D. H., Waugh, M. M., Ferrin, D. M., Alcantara, T., and Rasmussen, S. L. 2004. Infection and colonization of melon roots by Monosporascus cannonballus in two cropping seasons in Arizona and California. Plant Pathol. 53:54-57.

14. Stanghellini, M. E., Mathews, D. M., and Misaghi, I. J. 2010. Pathogenicity and management of Olpidium bornovanus, a root pathogen of melons. Plant Dis. 94:163-166.

15. Uecker, F. A., and Pollack, F. G. 1975. Development and cytology of Monosporascus cannonballus. Bot. Gaz. 136:333-340. 